\title{
Setting Reserve Requirements to Approximate the Efficiency of the Stochastic
} Dispatch

Dvorkin, Vladimir; Delikaraoglou, Stefanos; Morales, Juan Miguel

Published in:

IEEE Transactions on Power Systems

Link to article, DOI:

10.1109/TPWRS.2018.2878723

Publication date:

2018

Document Version

Peer reviewed version

Link back to DTU Orbit

Citation (APA):

Dvorkin, V., Delikaraoglou, S., \& Morales, J. M. (2018). Setting Reserve Requirements to Approximate the Efficiency of the Stochastic Dispatch. IEEE Transactions on Power Systems, 34(2), 1524 - 1536.

https://doi.org/10.1109/TPWRS.2018.2878723

\section{General rights}

Copyright and moral rights for the publications made accessible in the public portal are retained by the authors and/or other copyright owners and it is a condition of accessing publications that users recognise and abide by the legal requirements associated with these rights.

- Users may download and print one copy of any publication from the public portal for the purpose of private study or research.

- You may not further distribute the material or use it for any profit-making activity or commercial gain

- You may freely distribute the URL identifying the publication in the public portal 


\title{
Setting Reserve Requirements to Approximate the Efficiency of the Stochastic Dispatch
}

\author{
Vladimir Dvorkin Jr., Student member, IEEE, Stefanos Delikaraoglou, Member, IEEE, \\ and Juan M. Morales, Senior member, IEEE
}

\begin{abstract}
This paper deals with the problem of clearing sequential electricity markets under uncertainty. We consider the European approach, where reserves are traded separately from energy to meet exogenous reserve requirements. Recently proposed stochastic dispatch models that co-optimize these services provide the most efficient solution in terms of expected operating costs by computing reserve needs endogenously. However, these models are incompatible with existing market designs. This paper proposes a new method to compute reserve requirements that bring the outcome of sequential markets closer to the stochastic energy and reserves co-optimization in terms of cost efficiency. Our method is based on a stochastic bilevel program that implicitly improves the inter-temporal coordination of energy and reserve markets, but remains compatible with the European market design. We use two standard IEEE reliability test cases to illustrate the benefit of intelligently setting operating reserves in single and multiple reserve control zones.
\end{abstract}

Index Terms-Bilevel optimization, electricity markets, market clearing, reserve requirements, stochastic programming.

\section{NOMENCLATURE}

The main notation used in this paper is stated below. Additional symbols are defined in the paper where needed. All symbols are augmented by index $t$ when referring to different time periods.

\section{A. Sets and Indices}

$\Lambda \quad$ Set of transmission lines.

$\omega \in \Omega$ Set of wind power production scenarios.

$i \in I \quad$ Set of conventional generation units.

$j \in J \quad$ Set of loads.

$k \in K$ Set of wind power units.

$n \in N$ Set of nodes.

$z \in Z \quad$ Set of reserve control zones.

\{\}$_{n} \quad$ Mapping of \{\} into the set of nodes.

\{\}$_{z} \quad$ Mapping of \{\} into the set of reserve control zones.

V. Dvorkin Jr. is with the Technical University of Denmark, Kgs. Lyngby, Denmark (e-mail: vladvo@elektro.dtu.dk).

S. Delikaraoglou is with the ETH Zurich, Zurich, Switzerland (e-mail: delikaraoglou@eeh.ee.ethz.ch).

J.M.Morales is with the University of Malaga, Malaga, Spain (e-mail: juan.morales@uma.es).

The work by Vladimir Dvorkin Jr. was supported in part by the Russian Foundation for Basic Research (RFBR) according to the research project No. 16-36-00389. The work by Juan M. Morales was supported in part by the Spanish Ministry of Economy, Industry and Competitiveness through project ENE2017-83775-P; and in part by the European Research Council (ERC) under the EU Horizon 2020 research and innovation programme (grant agreement No. 755705) and the Research Program for Young Talented Researchers of the University of Malaga through project PPIT-UMA-B12017/18.

\section{B. Decision variables}

$\delta_{n}^{\text {DA }} \quad$ Day-ahead voltage angle at node $n$ [rad].

$\delta_{n \omega}^{R T} \quad$ Real-time voltage angle at node $n$ in scenario $\omega[\mathrm{rad}]$.

$D_{z}^{\mathrm{U} / \mathrm{D}} \quad \mathrm{Up}$-/Downward reserve requirement in zone $z[\mathrm{MW}]$.

$L_{j \omega}^{\text {sh }} \quad$ Shedding of load $j$ in scenario $\omega$ [MW].

$P_{i}^{\mathrm{C}} \quad$ Day-ahead dispatch of conventional unit $i[\mathrm{MW}]$.

$P_{k}^{\mathrm{W}} \quad$ Day-ahead dispatch of wind power unit $k$ [MW].

$P_{k \omega}^{\mathrm{W}, \mathrm{sp}} \quad$ Wind spillage of unit $k$ in scenario $\omega$ [MW].

$R_{i}^{\mathrm{U} / \mathrm{D}} \mathrm{Up}$-/Downward reserve provision from unit $i$ [MW].

$r_{i \omega}^{\mathrm{U} / \mathrm{D}} \quad \mathrm{Up}$-/Downward reserve deployment of unit $i$ in scenario $\omega[\mathrm{MW}]$.

\section{Parameters}

$\pi_{\omega} \quad$ Probability of occurrence of wind power production scenario $\omega$.

$C_{i} \quad$ Day-ahead price offer of unit $i$ [\$/MWh].

$C_{i}^{\mathrm{U} / \mathrm{D}} \quad \mathrm{Up}-/$ Downward reserve price offer of unit $i[\$ / \mathrm{MWh}$.

$C^{\text {VoLL }}$ Value of lost load [\$/MWh].

$\bar{F}_{n m} \quad$ Capacity of transmission line $(n, m)$ [MW].

$L_{j} \quad$ Demand of load $j$ [MWh].

$\bar{P}_{i} \quad$ Day-ahead quantity offer of unit $i$ [MW].

$\bar{R}_{i}^{\mathrm{U} / \mathrm{D}} \quad$ Up-/Downward reserve capacity offer of unit $i$ [MW].

$\widehat{W}_{k} \quad$ Expected generation of wind power unit $k$ [MW].

$W_{k \omega} \quad$ Wind power realization of unit $k$ in scenario $\omega$ [MW].

$X_{n m} \quad$ Reactance of transmission line $(n, m)$ [p.u.].

\section{INTRODUCTION}

$\mathbf{E}$ LECTRICITY markets are commonly organized in a sequence of trading floors in which different services are traded in various time-frames. According to the European market architecture, this sequence consists of reserve and day-ahead markets that are cleared 12-36 hours before actual power system operation and pertain to trading reserve capacity and energy services, respectively. Getting close to actual delivery of electricity, a real-time market is organized to balance deviations from the initial schedule. This market design has been established following a conventional view of power system operation, where uncertainty was induced by equipment contingencies or minor forecast errors of electricity demand. However, considering the increasing shares of renewable generation, this design has limited ability to cope with variable and uncertain energy sources, while maintaining a sufficient level of reliability at a reasonable cost [1].

To account for the uncertain nature of renewable generation, recent literature proposes economic dispatch models [2], [3] 
and unit commitment formulations [4]-[6] based on stochastic optimization. Unlike the conventional market design, which downplays the cost of uncertainty, the stochastic model makes use of a probabilistic description of uncertainty and dispatches the system accounting for plausible forecast errors. In this case, reserve requirements are computed endogenously, instead of relying on rule-of-thumb methods such as as the $\mathrm{N}$ 1 security criterion. Although the resulting stochastic ideal schedule provides the most efficient solution in terms of expected operating system costs, this design is not adopted in practice due to still unresolved issues like the violation of the least-cost merit-order principle [7].

There are several research contributions devoted to approximating the stochastic ideal solution, i.e., approaching the expected operating cost provided by the stochastic dispatch model while sidestepping its theoretical drawbacks, namely, the violation of cost recovery and revenue adequacy for certain realizations of the random variables. The cost recovery property guarantees that the profit of each conventional producer is greater than or equal to its operating costs. The revenue adequacy property requires that the payments that the system operator must make to and receive from the participants do not cause it to incur a financial deficit. Authors in [8] propose a new market-clearing procedure according to which wind power is dispatched to a value different than its forecast mean, such that the expected system cost is minimized. This procedure respects the merit order of the day-ahead market and thus ensures cost recovery of the flexible units. An enhanced stochastic dispatch that guarantees both cost recovery and revenue adequacy for every uncertainty realization is introduced in [9]. The main obstacle preventing the implementation of these two models is that they require changing the state of affairs of conventional market structures. Finally, authors in [10] propose a stochastic dispatch model that aims at generating proper price signals that incentivize generators to provide reliability services akin to reserves. This model also guarantees cost recovery and revenue adequacy for every uncertainty realization, but in the meantime it does also require significant changes in market design as well as in the offering strategies of the renewable power producers.

More in line with the current practices of the European market design, [11] proposes a systematic method to adjust available transfer capacities in order to bring operational efficiency of interconnected power systems closer to the stochastic solution. In the US electricity markets, several Independent System Operators (ISOs), e.g., the California ISO (CAISO) and Midcontinent ISO (MISO) are implementing new ramping capacity products to increase the ramping ability of the system during the real-time re-dispatch in order to cope with steep ramps of net load [12]. Essentially, these flexibility products aim to resemble the stochastic dispatch, which inherently finds the optimal allocation of flexible resources between energy and ramping services. In the same vein, several US ISOs, as for instance the New York ISO, the ISO New England, the MISO, and the Pennsylvania-New Jersey-Maryland (PJM) market, have introduced an operating reserve demand curve (ORDC) in their real-time market [13]. Motivated by the two-stage stochastic dispatch model, the ORDC mechanism adjusts electricity prices to reflect the scarcity value of reserves for the system operator and incentivize market players to dispatch their units according to a socially optimal schedule. The price adjustment through ORDC leads theoretically to perfect arbitrage between energy and reserves in case these two products are co-optimized [14]. However, in the European market that separates energy and reserve capacity trading this arbitrage is inefficient per se, since market players have to value reserves prior to the energy-only market clearing.

This paper proposes an alternative approach to approximate the stochastic ideal dispatch solution through an intelligent setting of zonal reserve requirements in sequentially cleared electricity markets akin to the European architecture. Here, we solely focus on operating reserves, i.e., generation that is dispatched to respond to net load variations based on economic bids, rather than on regulating services that are activated by automatic generation control. Traditionally, requirements for operating reserves are defined based on deterministic security criteria, such as N-1 security constraint violations, where reserves are dimensioned to cover the largest contingency in the system [15], or based on a mean forecast load error and forced outage rate of system components over a certain horizon, as in the PJM market [16]. The main drawback of those approaches is that they ignore the probabilistic nature of renewable generation and neglect the economic impact of reserve needs on subsequent operations. In order to account for the operational uncertainty, recent literature proposes reserve dimensioning methods based on probabilistic criteria, according to which reserve requirements are drawn from the probabilistic description of uncertainties [17]-[26]. For example, [17] suggests to define the reserve needs such that they cover $97.7 \%(3 \sigma)$ of the total variation of a Gaussian distribution modeling the joint wind-load uncertainty, disregarding the fact that wind power forecast errors are described by non-Gaussian distributions [24]. As a remedy to this drawback, [25] proposed a method for setting the reserve requirements using non-parametric probabilistic wind power forecasts. Flying brick and probability box methods in [20] and [21], respectively, compute robust envelopes that enclose the net load with a specified probability level. The recent extension of these methods called flexibility envelopes was suggested in [22]. These envelopes are based on the same principles but evolve in time to respect the temporal evolution of reserve requirements. As demonstrated in [20], [21] and [23], the probabilistic reserve concepts might be integrated into the actual energy management system and derive requirements for capacity, ramping capability and ramping duration of flexible units. In contrast to the deterministic practices, the benefit of these methods is that reserve requirements, drawn from accurately predicted distributions, minimize extreme balancing actions provoked by under- or over-procurement of reserves. However, probabilistic requirements are still an exogenous input to the power dispatch, which disregards their potential impact on expected cost.

To this end, we propose a model to determine reserves based on a stochastic bilevel programming problem, which provides the cost-optimal reserve quantities for a European-type market structure. In line with the stochastic dispatch mechanism, our 
model computes the reserve requirements that minimize the expected system cost, anticipating their projected impact on the subsequent operations. Additionally, these requirements are defined accounting for the actual decision-making process, i.e., the sequence of market-clearing procedures, zonal representation of the power network and the least-cost merit-order principle in all trading floors. As a result, the implementation of these requirements in a conventional market setting, results in a compromise solution between traditional reserve dimensioning practices and the stochastic dispatch model in terms of expected operating cost. Naturally, our approach has limitations: we consider a simplified market setup with a strictly convex representation. Nevertheless, our results do indicate that the intelligent setting of reserve requirements can enhance the short-run cost efficiency of the conventional market with large shares of renewable generation.

The proposed model can be used as an analytic tool to provide technical and economic insights about the efficacy of different reserve capacity quantification methods, while it can be also used as a decision-support tool by system operators during the reserve setting process. In the latter case, this model can be presumably executed before the day-ahead reserve capacity auction in order to define the reserve requirements that will be used as input in the actual market-clearing process. Nevertheless, the incorporation of this method in the operational strategy of the system operator does not entail any changes in the existing market setup, since the model output is solely under the discretion of the system operator and decoupled from market operations.

The reminder of this paper is organized as follows. Section III describes the conventional market design and its counterfactual stochastic representation. Section $[\mathrm{III}$ introduces the proposed stochastic bilevel programming problem to compute the optimal reserve requirements that approximate the ideal stochastic solution maintaining the sequential market structure. Section IV] explains the solution strategy based on the multicut Bender's algorithm for large-scale applications. Section V provides applications of the proposed model to the IEEE-24 and IEEE-96 reliability test systems. Section VI concludes the paper.

\section{ELECTRICITY MARKET CLEARING MODELS}

In this section, we first describe the conventional market structure and the stochastic dispatch model. We then introduce the necessary modeling assumptions and provide the mathematical formulations of both models.

\section{A. Conventional market and stochastic dispatch framework}

In Europe, power markets are cleared in sequential and independent auctions which can be represented by the simplified decision-making process illustrated in Fig. 1(a), which is referred to as the conventional market-clearing model. First, the system operator defines zonal reserve requirements $\mathcal{D}$ based on certain security standards. Then, the reserve capacity market is cleared based on the offer prices and quantities submitted by the flexible producers to find the optimal upward and downward reserve allocation $\Phi^{\mathrm{R}^{*}}$ that minimizes reserve

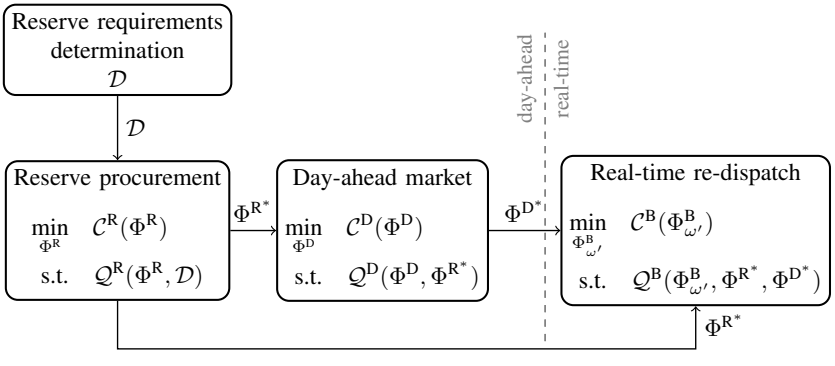

(a) Conventional market-clearing model

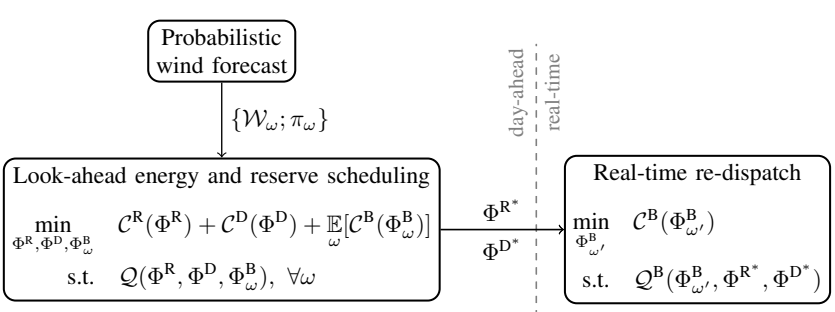

(b) Stochastic dispatch model.

Fig. 1. Decision sequences in conventional (a) and stochastic (b) dispatch models.

procurement costs $\mathcal{C}^{\mathrm{R}}$. This allocation accounts for upward and downward reserve requirement constraints included in the set $\mathcal{Q}^{\mathrm{R}}$. At the next stage, power producers submit their price-quantity offers to the day-ahead market that provides the optimal energy schedule $\Phi^{\mathrm{D}^{*}}$ that minimizes the day-ahead energy cost $\mathcal{C}^{\mathrm{D}}$. The set of day-ahead market constraints $\mathcal{Q}^{\mathrm{D}}$ takes into account the reserve capacity $\Phi^{\mathrm{R}^{*}}$ procured at the previous stage. Closer to delivery time, when realization of uncertainty $\omega^{\prime}$ is known, the system operator runs the real-time market to define a set of optimal re-dispatch actions $\Phi_{\omega^{\prime}}^{\mathrm{B}}$ that minimizes the balancing $\operatorname{cost} \mathcal{C}^{\mathrm{B}}$, considering the previously procured reserve $\Phi^{\mathrm{R}^{*}}$. In this conventional market design, the choice of reserve requirements $\mathcal{D}$ has a direct impact on the total expected system cost. In fact, the choice of $\mathcal{D}$ influences reserve procurement decisions $\Phi^{\mathrm{R}}$, which in turn affect dayahead $\Phi^{\mathrm{D}}$ and real-time $\Phi^{\mathrm{B}}$ energy dispatch decisions.

An alternative model for reserves and energy scheduling is the stochastic dispatch model outlined in Fig. 1(b) This is a two-stage stochastic programming model in which firststage decisions pertain to reserve procurement and day-ahead energy schedule, whereas the second stage models the recourse actions that restore power balance during real-time operation. The stochastic dispatch model takes as input a probabilistic wind power forecast in the form of a scenario set $\Omega$ and endogenously computes reserve needs. This way, it naturally coordinates all trading floors by co-optimizing reserve $\left(\Phi^{\mathrm{R}}\right)$ and energy $\left(\Phi^{\mathrm{D}}\right)$ schedules, anticipating their impact on the subsequent expected balancing cost $\mathbb{E}\left[\mathcal{C}^{\mathrm{B}}\left(\Phi_{\omega}^{\mathrm{B}}\right)\right]$ estimated over the scenario set $\Omega$. It should be noted that the co-optimization of reserve procurement and energy schedules is a requirement for the implementation of this ideal coordination between the different trading floors.

In the stochastic dispatch, reserve requirements are a byproduct of the energy and reserve co-optimization problem, resulting in the most efficient solution in terms of total expected operating cost. Moreover, unlike the conventional 
market model that schedules reserve and day-ahead energy quantities according to the least-cost merit-order principle, the stochastic model schedules generation capacity accounting for potential network congestion during real-time operations, which may lead to expensive balancing actions [2]. This way generators may be scheduled out-of-merit, i.e., more expensive units are dispatched over less expensive ones, in order to minimize the expected costs.

Despite its superiority in terms of cost efficiency, the stochastic model suffers from several drawbacks preventing its practical implementation. As already mentioned, the violation of the merit-order principle results in cost recovery and revenue adequacy only in expectation, while for some uncertainty realizations these two essential economic properties may not hold [2]. This issue disputes the well-functioning of electricity markets in long term, since flexible producers may end up in loss-making positions in one or more scenarios, despite the fact that their expected profit is non-negative. Therefore, these market participants may opt out of the shortrun electricity markets or even be discouraged to perform new investments if they are exposed to significant financial risks. In the meantime, the fact that revenue adequacy is only guaranteed in expectation exposes the market operator to the risk of financial deficit. Therefore, a realistic implementation of this market model would require the establishment of outof-the-market mechanisms, akin to the uplift payments used in the US markets, to provide an ex-post compensation of potential economic deficits. In view of this practical caveats, we do not foresee an actual market clearing implementation of the stochastic dispatch model. Moreover, the co-optimization of day-ahead energy and capacity reserve markets is not compatible with the European market structure, which dictates that the trading of reserves and energy products is organized in independent sequential auctions. However, in this work, we show that the stochastic dispatch solution can be approximated in the conventional market-clearing model by intelligently setting the reserve requirements $\mathcal{D}$, sidestepping the drawbacks of the stochastic model and improving the efficiency of the existing market setup.

\section{B. Modeling assumptions}

We use the following set of assumptions to derive computationally tractable yet sensible formulations of the different dispatch models. Following the European practice, we consider a zonal representation of the network for reserve procurement. In an attempt to build a more generic model, the network topology is included in the day-ahead and real-time dispatch models considering a DC approximation of power flows. Reserve and energy supply functions are linear, and all generators are considered to behave as price takers. System loads are inelastic with a large value of lost load. This way, the maximization of the social welfare is equivalent to cost minimization. Flexible units deploy operating reserves with marginal costs of production. The incentive to provide flexibility services is accounted for in reserve offering prices. Following the prevailing portfolio bidding adopted in the European markets [27], we consider that all unit commitment and inter-temporal constraints are integrated into the bidding strategies of the generating units. For instance, the commitment of thermal units in practice might be controlled by market participants when offering at either zero price or market price cap. Similarly, offering a part of capacity at zero and even negative price ensures the compliance with the technical minimum constraint of thermal units. This approach is compatible with the European market structure and preserves the convexity of the reserve capacity and day-ahead market-clearing algorithms. In principle, the proposed model can be also applied to market designs that involve non-convex constraints, as for instance the majority of electricity markets in the US, using tight convex relaxations of the unit commitment binary variables. However, this approach lies out of scope of this paper, but we refer the interested reader to [28], [29] for further discussion. Finally, uncertainty is described by a finite set of scenarios and solely induced by stochastic wind power production.

\section{Mathematical formulation}

1) Conventional market-clearing model: The sequential procedure, sketched in Fig. 1(a), for each hour of the next day is modeled by the following three linear optimization problems.

The reserve procurement problem writes as:

$$
\begin{array}{ll}
\min _{\Xi^{\mathrm{OR}}} & \sum_{i \in I}\left(C_{i}^{\mathrm{U}} R_{i}^{\mathrm{U}}+C_{i}^{\mathrm{D}} R_{i}^{\mathrm{D}}\right) \\
\text { s.t. } & \sum_{i \in I_{z}} R_{i}^{\mathrm{U}}=D_{z}^{\mathrm{U}}, \quad \sum_{i \in I_{z}} R_{i}^{\mathrm{D}}=D_{z}^{\mathrm{D}}, \quad \forall z \in Z, \\
& R_{i}^{\mathrm{U}}+R_{i}^{\mathrm{D}} \leq \bar{P}_{i}, \quad \forall i \in I, \\
& 0 \leq R_{i}^{\mathrm{U}} \leq \bar{R}_{i}^{\mathrm{U}}, \quad 0 \leq R_{i}^{\mathrm{D}} \leq \bar{R}_{i}^{\mathrm{D}}, \quad \forall i \in I,
\end{array}
$$

where $\Xi^{\mathrm{OR}}=\left\{R_{i}^{\mathrm{U}}, R_{i}^{\mathrm{D}}, \forall i\right\}$ is the set of optimization variables comprising the upward and downward reserve schedule per each flexible generator. Optimal $\Xi^{\mathrm{OR} *}$ minimizes the reserve procurement cost given by (1a). Equality constraints (1b) ensure that zonal reserve upward and downward requirements, denoted as $D_{z}^{\mathrm{U}}$ and $D_{z}^{\mathrm{D}}$, respectively, are fulfilled, whereas inequality constraints (1c) - (1d) account for the quantity offers of each flexible generator.

Once reserve allocation $\left\{R_{i}^{\mathrm{U} *}, R_{i}^{\mathrm{D} *}, \forall i\right\}$ is determined, the least-cost day-ahead energy schedule is computed solving the following optimization problem:

$$
\begin{array}{ll}
\min _{\Xi^{\mathrm{DA}}} & \sum_{i \in I} C_{i} P_{i}^{\mathrm{C}} \\
\text { s.t. } & \sum_{i \in I_{n}} P_{i}^{\mathrm{C}}+\sum_{k \in K_{n}} P_{k}^{\mathrm{W}}-\sum_{j \in J_{n}} L_{j} \\
& -\sum_{m:(n, m) \in \Lambda} \frac{\delta_{n}^{\mathrm{DA}}-\delta_{m}^{\mathrm{DA}}}{x_{n m}}=0, \quad \forall n \in N, \\
& R_{i}^{\mathrm{D} *} \leq P_{i}^{\mathrm{C}} \leq \bar{P}_{i}-R_{i}^{\mathrm{U} *}, \quad \forall i \in I, \\
& 0 \leq P_{k}^{\mathrm{W}} \leq \widehat{W}_{k}, \quad \forall k \in K, \\
& \frac{\delta_{n}^{\mathrm{DA}}-\delta_{m}^{\mathrm{DA}}}{x_{n m}} \leq \bar{F}_{n m}, \quad \forall(n, m) \in \Lambda,
\end{array}
$$

where $\Xi^{\mathrm{DA}}=\left\{P_{i}^{\mathrm{C}}, \forall i ; P_{k}^{\mathrm{W}}, \forall k ; \delta_{n}^{\mathrm{DA}}, \forall n\right\}$ is the set of variables including day-ahead energy quantities for each conventional 
and stochastic generator as well as voltage angles at each node. The objective function $2 \mathrm{a}$ to be minimized is the dayahead energy cost, subject to nodal power balance constraints (2b), offering limits of conventional and stochastic generators (2c)-2d) and transmission capacity limits (2e). Note that the reserve procurement decisions from the previous stage limit the dispatch of flexible generators at the day-ahead stage. In this design, stochastic production is bounded by the conditional expectation $\widehat{W}_{k}$.

Getting closer to real-time operation, any deviation from the optimal day-ahead dispatch $\left\{P_{i}^{\mathrm{C} *}, \forall i ; P_{k}^{\mathrm{W} *}, \forall k ; \delta_{n}^{\mathrm{DA} *}, \forall n\right\}$ has to be covered by proper balancing actions. For a specific realization of stochastic production $W_{k \omega^{\prime}}$, the optimal redispatch is found solving the following linear programming problem:

$$
\begin{aligned}
& \min _{\Xi^{\mathrm{RT}}} \sum_{i \in I} C_{i}\left(r_{i \omega^{\prime}}^{\mathrm{U}}-r_{i \omega^{\prime}}^{\mathrm{D}}\right)+\sum_{j \in J} C^{\mathrm{VoLL}} L_{j \omega^{\prime}}^{\mathrm{sh}} \\
& \text { s.t. } \sum_{i \in I_{n}}\left(r_{i \omega^{\prime}}^{\mathrm{U}}-r_{i \omega^{\prime}}^{\mathrm{D}}\right)+\sum_{k \in K_{n}}\left(W_{k \omega^{\prime}}-P_{k}^{\mathrm{W}^{*}}-P_{k \omega^{\prime}}^{\mathrm{W}, \mathrm{sp}}\right) \\
&+\sum_{j \in J_{n}} L_{j \omega^{\prime}}^{\mathrm{sh}}-\sum_{m:(n, m) \in \Lambda} \frac{\delta_{n \omega^{\prime}}^{\mathrm{RT}}-\delta_{n}^{\mathrm{DA}^{*}}-\delta_{m \omega^{\prime}}^{\mathrm{RT}}+\delta_{m}^{\mathrm{DA}}}{x_{n m}} \\
&=0, \quad \forall n \in N, \\
& 0 \leq r_{i \omega^{\prime}}^{\mathrm{U}} \leq R_{i}^{\mathrm{U} *}, \quad 0 \leq r_{i \omega^{\prime}}^{\mathrm{D}} \leq R_{i}^{\mathrm{D} *}, \quad \forall i \in I \\
& \frac{\delta_{n \omega^{\prime}}^{\mathrm{RT}}-\delta_{m \omega^{\prime}}^{\mathrm{RT}} \leq \bar{F}_{n m}, \quad \forall(n, m) \in \Lambda}{x_{n m}} \\
& 0 \leq P_{k \omega^{\prime}}^{\mathrm{W}, \mathrm{sp}} \leq W_{k \omega^{\prime}}, \quad \forall k \in K \\
& 0 \leq L_{j \omega^{\prime}}^{\mathrm{sh}} \leq L_{j}, \quad \forall j \in J
\end{aligned}
$$

where $\Xi^{\mathrm{RT}}=\left\{r_{i \omega^{\prime}}^{\mathrm{U}}, r_{i \omega^{\prime}}^{\mathrm{D}}, \forall i ; L_{j \omega^{\prime}}^{\mathrm{sh}}, \forall j ; P_{k \omega^{\prime}}^{\mathrm{W}, \mathrm{sp}}, \forall k ; \delta_{n \omega^{\prime}}^{\mathrm{RT}}, \forall n\right\}$ is the set of re-dispatch decisions, comprising activation of operating reserves, load shedding, wind spillage and real-time voltage angles. The objective function (3a) to be minimized is the balancing cost. Equality constraints (3b) ensure the realtime nodal power balance. Inequalities (3c) limit activation of upward and downward reserves considering the procured reserve quantities. Constraints (3d) account for the power capacity of transmission lines. Finally, inequalities (3e) and (3f) limit wind spillage and load shedding actions to the actual realization of production and system demand, respectively.

2) Stochastic dispatch model: Assuming that wind power uncertainty is described by a finite set of outcomes $W_{k \omega}$ with corresponding probabilities $\pi_{\omega}$, the stochastic dispatch procedure outlined in Fig. 1(b) writes as follows:

$$
\begin{aligned}
& \min _{\Xi^{\mathrm{SD}}} \sum_{i \in I}\left(C_{i}^{\mathrm{U}} R_{i}^{\mathrm{U}}+C_{i}^{\mathrm{D}} R_{i}^{\mathrm{D}}+C_{i} P_{i}^{\mathrm{C}}\right)+ \\
& \sum_{\omega} \pi_{\omega}\left(\sum_{i \in I} C_{i}\left(r_{i \omega}^{\mathrm{U}}-r_{i \omega}^{\mathrm{D}}\right)+\sum_{j \in J} C^{\mathrm{VoLL}} L_{j \omega}^{\mathrm{sh}}\right) \\
& \text { s.t. constraints (1b) - (1d) } \\
& \text { constraints 2b - 2e } \\
& \text { constraints (3b) }-(3 \mathrm{f}), \quad \forall \omega \in \Omega
\end{aligned}
$$

where $\Xi^{\mathrm{SD}}=\left\{\Xi^{\mathrm{OR}} \cup \Xi^{\mathrm{DA}} \cup \Xi^{\mathrm{RT}}, \forall \omega \cup\left(D^{\mathrm{U}}, D^{\mathrm{D}}\right)\right\}$ is the set of stochastic dispatch variables. The objective function (4a) to be minimized is the reserve and day-ahead energy cost as well as the expectation of the real-time cost, i.e., the expected cost over the entire decision sequence. Note, that upward and downward reserve requirements $D_{z}^{\mathrm{U}}$ and $D_{z}^{\mathrm{D}}$ in (1b) are decision variables and only used to reveal optimal reserve requirements in a stochastic programming sense.

After the optimal reserve procurement and day-ahead energy schedule are obtained, the system operator solves the real-time re-dispatch problem for a specific realization of the stochastic production $\omega^{\prime}$ using formulation $(3)$.

\section{APPROXIMATING THE STOCHASTIC IDEAL}

On the one hand, the conventional procedure has limited capability to accommodate large shares of stochastic production in a cost efficient manner compared to the stochastic dispatch. On the other hand, the adoption of the stochastic procedure appears to be unrealistic because it does not guarantee revenue adequacy and cost recovery for every uncertainty realization; these are important properties that, in contrast, hold in the sequential market structure [2], [8]. For this reason, our motivation is to enhance the cost-efficiency of the conventional market-clearing procedure without changing the market structure. In this line, we introduce a model that approximates the ideal stochastic solution within the conventional dispatch model by the appropriate setting of zonal reserve requirements. In essence, we aim at finding the reserve requirements that plugged into the conventional market-clearing model (1)-(3) will yield the minimum total expected system cost. To compute them, we use the bilevel programming problem illustrated in Fig. 2

This model comprises two levels. The objective function of the upper level is the same as (4a) in the stochastic model (4) and aims at minimizing the total expected system cost. The upper-level constraints enforce real-time re-dispatch limits. The lower level consists of two optimization problems, namely, the reserve procurement and day-ahead market clearing problems, which are identical to the corresponding optimization problems (1) and 2) of the conventional model. However, in this bilevel structure, reserve requirements $\mathcal{D}$ are decision variables of the upper-level problem, entering as parameters in the lower-level reserve procurement problem. Hence, reserve requirements $\mathcal{D}$ are not an exogenous input to this model but are internally optimized, accounting for their impact in all three trading floors. As shown in Fig. 2, the upper-level decision on $\mathcal{D}$ affects the reserve procurement schedule in the first lower-level problem, which in turn impacts the day-ahead clearing obtained from the second lower-level problem. In addition, the reserve and energy schedules $\Phi^{\mathrm{R}}$ and $\Phi^{\mathrm{D}}$ enter the upper level, constraining the real-time re-dispatch decisions.

The structure of this stochastic bilevel model guarantees that the temporal sequence of the different markets follows the existing European paradigm. Having the reserve capacity and day-ahead market clearings as two independent lower-level problems, ensures that reserves and day-ahead schedules are optimized separately, i.e., there is no co-optimization of energy and reserves, while none of these markets have information about the future re-dispatch actions. This property suffices to reproduce the real-time re-dispatch for each scenario indepen- 
dently by including the corresponding constraints only in the upper-level problem.

Compared to the stochastic model, the main advantage of this bilevel scheme is that it respects the merit-order principle in the reserve capacity and day-ahead energy markets. In fact, given the same reserve requirements, the solutions of both lower-level problems are identical to the solutions of problems (1) and (2). Nonetheless, the upper-level problem can still anticipate the impact of reserve requirements on all trading floors and consequently on the total expected cost.

Since this model is solved prior to any market-clearing procedure, we assume that the system operator can gather information on the price-quantity offers of market participants. Even in the case of having to use an estimation of pricequantity offers similar to the ORDC mechanism, our approach accounts systematically for the impact of reserve procurement and the structure of forecast errors in all three trading floors. In a more realistic setup, this information can be obtained using inverse optimization techniques as proposed in [30] and [31].

Mathematically, the proposed reserve determination model writes as the following stochastic bilevel programming problem:

$$
\begin{aligned}
& \min _{\Xi^{\mathrm{RT}}, D_{z}^{\mathrm{U}}, D_{z}^{\mathrm{D}}} \\
& \text { s.t. constraints 3b - (3f), } \forall \omega \in \Omega \text {, } \\
& D_{z}^{\mathrm{U}}, D_{z}^{\mathrm{D}} \geq 0, \quad \forall z \in Z, \\
& \left(R_{i}^{\mathrm{U}}, R_{i}^{\mathrm{D}}\right) \in \arg \left\{\begin{array}{ll}
\min _{\Xi^{\mathrm{OR}}} & 1 \mathrm{a} \\
\text { s.t. } & \text { constraints }(1 \mathrm{~b})-1 \mathrm{1d}]
\end{array}\right\}, \\
& \left(\begin{array}{c}
P_{i}^{\mathrm{C}}, P_{k}^{\mathrm{W}}, \\
\delta_{n}^{\mathrm{DA}}
\end{array}\right) \in \arg \left\{\begin{array}{l}
\min _{\Xi^{\mathrm{DA}}} \quad 2 \mathrm{a} \\
\text { s.t. } \\
\text { constraints } 2 \mathrm{~b}-2 \mathrm{2}]
\end{array}\right\} .
\end{aligned}
$$

According to the mathematical structure of model (5), the lower-level problems (5d) and (5e) guarantee that the reserve capacity and day-ahead energy markets are serially and independently optimized. This property is in accordance with the time-line of these trading floors in the European market framework. This temporal sequence is accomplished considering that upward $R_{i}^{\mathrm{U}^{*}}$ and downward $R_{i}^{\mathrm{D}^{*}}$ reserve schedules are variables of the reserve capacity market (5d) but enter as parameters in the day-ahead energy market $5 \mathrm{E}$. Moreover, neither problem (5d) nor (5e) can foresee the outcome of the balancing market, which is included in the upper level of model (5). As a result, both markets have no information about the effect of their decisions on the realtime market. In turn, constraints $(5 \mathrm{~b})-(5 \mathrm{c})$ and the third term of the objective function (4a) clear the real-time market of the conventional model (11)-(3), independently for each scenario $\omega \in \Omega$, considering that the real-time re-dispatch cannot impact the previous trading floors which are 'fixed' to the conventional market solution through the lower-level problems (5d) and 5e.

This formulation is computationally intractable, since it consists of an upper-level optimization problem constrained by two lower-level optimization problems. However, since both lower-level problems are convex with linear objective functions and constraints, they can be replaced by their Karush-

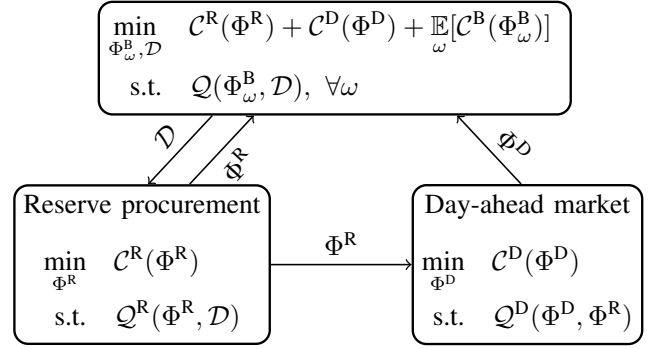

Fig. 2. Bilevel structure of the proposed reserve determination model.

Kuhn-Tucker optimally conditions, such that the problem can be recast as as a single-level mathematical program with equilibrium constraints (MPEC). The resulting model includes a set of nonlinear complementary slackness constraints, which can be linearized using disjunctive constraints or SOS1 variables, transforming the MPEC problem into a mixed-integer linear program (MILP) [32].

\section{SOLUTION STRATEGY}

The set of integer variables used to linearize the complementarity constraints of the lower-level problems (5d) and (5e) limits the application of the proposed reserve quantification model to power systems of moderate scale. For the large-scale applications, we propose an iterative solution strategy based on the multi-cut Bender's algorithm [33]. For a fixed reserve and day-ahead dispatch, the set of real-time constraints $3 \mathrm{~b}$ - (3f) is independent per scenario. This allows for Bender's decomposition where each subproblem solves a scenariospecific real-time re-dispatch problem. The subproblems at iteration $\nu$ write as follows:

$$
\begin{aligned}
\left\{\min _{\Xi_{s}^{\mathrm{RT}, \mathrm{B}}}\right. & C_{\omega}^{\mathrm{RT}(\nu)}:=\sum_{i \in I} C_{i}\left(r_{i \omega}^{\mathrm{U}}-r_{i \omega}^{\mathrm{D}}\right)+\sum_{j \in J} C^{\mathrm{VoLL}} L_{j \omega}^{\mathrm{sh}} \\
\text { s.t. } & R_{i}^{\mathrm{U}}=\tilde{R}_{i}^{\mathrm{U}(\nu)} \quad: \theta_{i \omega}^{R_{i}^{\mathrm{U}}(\nu)}, \quad \forall i \in I, \\
& R_{i}^{\mathrm{D}}=\tilde{R}_{i}^{\mathrm{D}(\nu)} \quad: \theta_{i \omega}^{R_{i}^{\mathrm{D}}(\nu)}, \quad \forall i \in I, \\
& P_{k}^{\mathrm{W}}=\tilde{P}_{k}^{\mathrm{W}(\nu)} \quad: \theta_{k \omega}^{P_{k}^{\mathrm{W}}(\nu)}, \quad \forall k \in K, \\
& \delta_{n}^{\mathrm{DA}}=\tilde{\delta}_{n}^{\mathrm{DA}(\nu)} \quad: \theta_{n \omega}^{\delta_{n}^{\mathrm{D}}(\nu)}, \quad \forall n \in N, \\
& \text { constraints [3b }- \text { 3f] }
\end{aligned}
$$

where $\Xi_{s}^{\mathrm{RT}, \mathrm{B}}=\Xi^{\mathrm{RT}} \cup\left\{R_{i}^{\mathrm{U}}, R_{i}^{\mathrm{D}}, \forall i ; P_{k}^{\mathrm{W}}, \forall k ; \delta_{n}^{\mathrm{DA}}, \forall n\right\}$ is the set of decision variables of each subproblem of the Bender's algorithm. Constraints (6b) - 6e fix the first-stage decisions to their optimal values obtained at the previous iteration, and the corresponding dual variables yield sensitivities of the reserve and day-ahead decisions used in Bender's cuts.

The master problem of the Bender's algorithm at iteration $\nu$ writes as follows:

$$
\begin{array}{ll}
\min _{\Xi^{\mathrm{M}, \mathrm{B}}} & \sum_{i \in I}\left(C_{i}^{\mathrm{U}} R_{i}^{\mathrm{U}}+C_{i}^{\mathrm{D}} R_{i}^{\mathrm{D}}+C_{i} P_{i}^{\mathrm{C}}\right)+\sum_{\omega \in \Omega} \pi_{\omega} \alpha_{\omega}^{(\nu)} \\
\text { s.t. } & \alpha_{\omega}^{(\nu)} \geq C_{\omega}^{\mathrm{RT}(\rho)}+\sum_{i \in I} \theta_{i \omega}^{R_{i}^{\mathrm{U}}(\rho)}\left(R_{i}^{\mathrm{U}}-R_{i}^{\mathrm{U}(\rho)}\right)
\end{array}
$$




$$
\begin{aligned}
& +\sum_{i \in I} \theta_{i \omega}^{R_{i}^{\mathrm{D}}(\rho)}\left(R_{i}^{\mathrm{D}}-R_{i}^{\mathrm{D}(\rho)}\right) \\
& +\sum_{k \in K} \theta_{k \omega}^{P_{k}^{\mathrm{W}}(\rho)}\left(P_{k}^{\mathrm{W}}-P_{k}^{\mathrm{W}(\rho)}\right) \\
& +\sum_{n \in N} \theta_{n \omega}^{\delta_{n}^{\mathrm{DA}}(\rho)}\left(\delta_{n}^{\mathrm{DA}}-\delta_{n}^{\mathrm{DA}(\rho)}\right), \\
& \rho=1 \ldots \nu-1, \forall \omega \in \Omega, \\
& \alpha_{\omega}^{(\nu)} \geq \underline{\alpha}, \quad \forall \omega \in \Omega, \\
& D_{z}^{\mathrm{U}}, D_{z}^{\mathrm{D}} \geq 0, \quad \forall z \in Z,
\end{aligned}
$$

where $\Xi^{\mathrm{M}, \mathrm{B}}=\Xi^{\mathrm{OR}} \cup \Xi^{\mathrm{DA}} \cup \alpha_{\omega}$ is the set of decisions variables of the master problem, and index $\rho$ is used to integrate the fixed values of the corresponding variables at previous iterations. The Bender's cuts are updated at each iteration by (7b) using sensitivities from all previous iterations, while $\sqrt{7 \mathrm{c}}$ imposes a lower bound $\underline{\alpha}$ on the auxiliary variable $\alpha$. Since the subproblems allow for load shedding, they are always feasible, requiring no feasibility cuts in the master problem. The algorithm converges at iteration $\nu$ if $\left|\sum_{\omega \in \Omega} \pi_{\omega}\left(\alpha_{\omega}^{(\nu)}-C_{\omega}^{\mathrm{RT}(\nu)}\right)\right| \leq \epsilon$, where $\epsilon$ is a predefined tolerance.

\section{Case Study}

In this section, we first describe the test system in Section $\mathrm{V}-\mathrm{A}$. In Section $\mathrm{V}-\mathrm{B}$ and Section $\mathrm{V}-\mathrm{C}$ we study the impact of reserve requirements on expected operating costs and we assess the remaining efficiency gap of our model with respect to the stochastic solution for a single reserve control zone. In Section V-D we extend our analysis to the case of multiple reserve control zones. In Section V-E we assess the model's performance in the presence of non-convex technical constraints. Finally, in Section V-F we demonstrate the scalability of the model using the proposed Bender's decomposition algorithm.

\section{A. Description of the test system}

To assess the performance of the different reserve determination models, a modified version of the IEEE 24-Bus RTS [34] is employed. The system consists of 34 transmission lines, 17 loads and 12 conventional generation units. The total generation capacity amounts to $3,375 \mathrm{MW}$, from which $1,100 \mathrm{MW}$ is flexible generation that can provide upward and downward reserves. We set upward reserve capacity price offers to be $30 \%$ of the marginal costs. Price offers for downward reserve capacity price offers are selected such that they compensate for the potential financial deficit induced by a loss-making position in the day-ahead market. We should note that this is only a heuristic approach to address the possibility that some flexible producers incur financial losses due to their combined positions in the reserve capacity and day-ahead energy markets. This situation may emerge if the downward reserve capacity $R_{i}^{D *}$ awarded to a generator, and in turn imposed as a lower bound in the day-ahead market constraint (2c), forces this unit to produce even if the day-ahead energy price is lower than its marginal production cost. This pitfall results from the separation of reserve capacity and energy markets in the European framework. In turn, the physical coupling of these two products is accounted for internally in the trading strategies of the market participants when they submit their price-quantity offers in the corresponding markets according to their risk appetite. A detailed study of this issue constitutes a separate research topic and lies out of the scope of this work, but the interested reader is referred to [35] and [36] for further information on this topic. Apart from conventional generators, there are six wind farms bidding at zero marginal cost and sited as explained in [34]. We consider a 24-hour load profile with a peak value of 2,650 MW obtained from [34]. The loads are assumed to be inelastic with the value of lost load equal to $\$ 500 / \mathrm{MW}$ for all operating hours. The relevant GAMS codes and simulation data are provided in the electronic companion of the paper [37].

All simulations are carried out using a standard PC with Intel Core i5 CPU with a clock rate of $2.7 \mathrm{GHz}$ requiring no more than $8 \mathrm{~GB}$ of RAM. The CPU time required to solve the conventional model (1)-(3), stochastic model (4) and bilevel model (5) in Sections $\bar{V}-\mathrm{B}, \mathrm{V}-\mathrm{D}$ is kept below 30s when solving per operating hour. The sequential market with unit commitment and inter-temporal constraints is solved in less than a minute in Section V-E The CPU time corresponding to the last case study is reported separately in Section $\mathrm{V}-\mathrm{F}$

\section{B. Impact of reserve requirements on expected system cost}

In this section we assess the expected cost of operating the power system under the conventional market setup (1)-(3), when this is fed with the reserve requirements determined by different approaches for reserve dimensioning, including our proposal. To this end, we consider the time period corresponding to the peak-load hour. Besides, the capacity of each wind power farm is set to $100 \mathrm{MW}$. Next we discuss the results linked to each reserve dimensioning approach:

1) The probabilistic approach defines the reserve requirements from the predictive cumulative distribution function (CDF) $F$ of the total wind power portfolio, as the distance between the expected wind power production $\widehat{W}$ and a specified quantile $q^{(\alpha)}=F^{-1}(\alpha)$ with nominal proportion $\alpha \in[0,1]$. This approach resembles the stateof-the-art reserve-dimensioning processes employed by European system operators using probabilistic forecast information [26]. For a reliability level $\xi=\bar{\alpha}-\underline{\alpha}$, the upward and downward reserve needs are dimensioned as follows:

$$
\begin{aligned}
& D^{\mathrm{U}}=\widehat{W}-F^{-1}(\bar{\alpha}), \\
& D^{\mathrm{D}}=F^{-1}(\underline{\alpha})-\widehat{W} .
\end{aligned}
$$

We initially consider $\underline{\alpha}=5 \%$ and $\bar{\alpha}=1-\underline{\alpha}=95 \%$ corresponding to a reliability level $\xi=90 \%$. The resulting requirements amount to $127.9 \mathrm{MW}$ and $89.1 \mathrm{MW}$ for upward and downward reserves, respectively. 


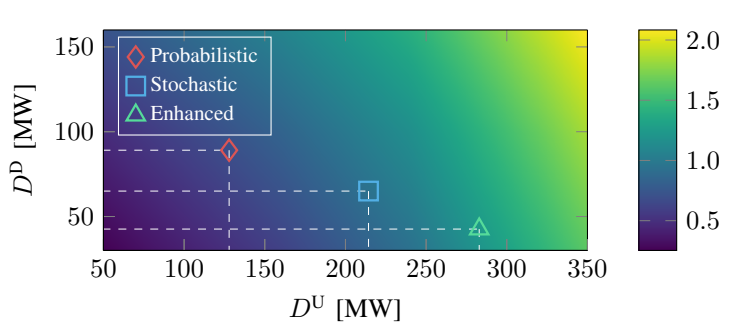

(a) Reserve procurement cost

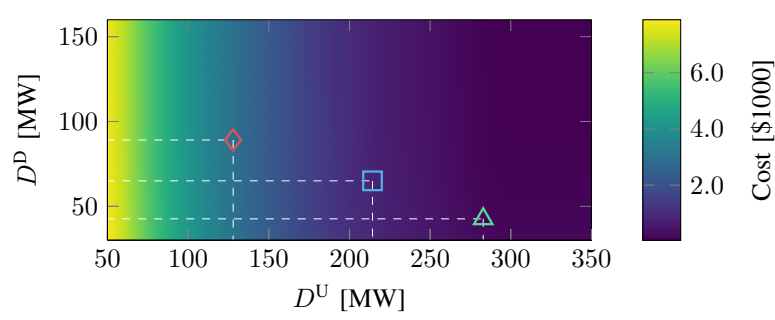

(c) Expected re-dispatch cost

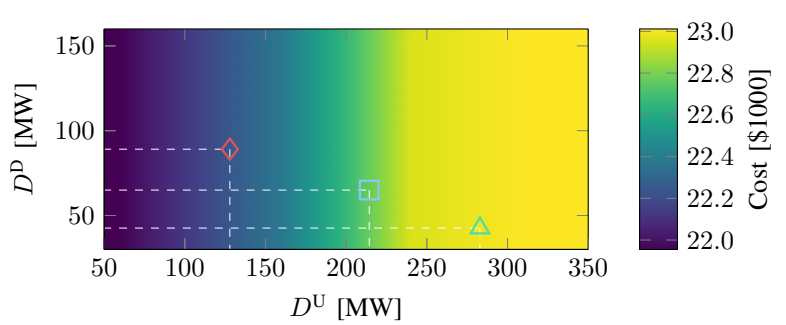

(b) Day-ahead energy cost

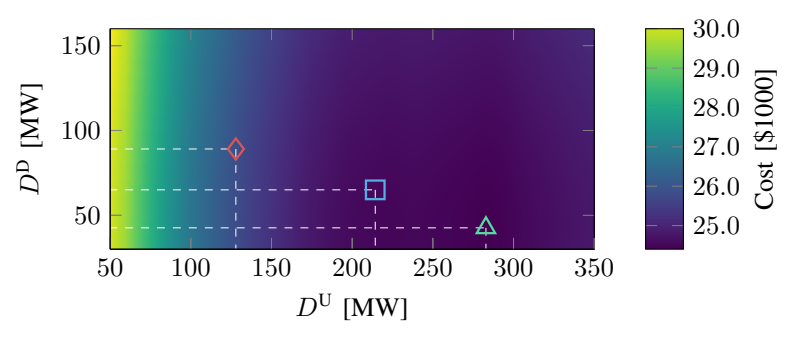

(d) Expected total system cost

Fig. 3. Impact of downward $D^{\mathrm{D}}$ and upward $D^{\mathrm{U}}$ operating reserve requirements on the reserve (a), day-ahead (b), expected re-dispatch (c) and expected total (d) costs in the conventional procedure (1)-3. The color density indicates the cost at the considered trading floor.

2) The stochastic approach derives the reserve requirements from the stochastic dispatch model (4). These requirements are equal to $214.3 \mathrm{MW}$ for upward and 65.0 MW for downward reserves, respectively.

3) The enhanced approach computes the reserve requirements using the proposed reserve determination model (5). Resulting reserve needs amount to $282.9 \mathrm{MW}$ and 42.6 MW for upward and downward reserves, respectively.

The expected total system costs resulting from the implementation of the probabilistic, stochastic and enhanced operating reserve approaches are $\$ 25,890, \$ 24,531$ and $\$ 24,408$, respectively. The total cost break-down is shown in Fig. 3 . which demonstrates the impact of the reserve requirements on the cost of the different trading floors in the conventional dispatch procedure. Figure $3(\mathrm{a})$ shows that the reserve needs computed using the proposed model result in the highest reserve procurement cost among the different approaches, mainly due to a larger volume of upward reserve provision. In turn, efficient flexible generation that could be scheduled in the day-ahead market is now set aside to provide upward reserves. Considering that the price offers for upward reserve are proportional to the day-ahead price offers, the withdrawal of these resources increases the day-ahead energy cost, as shown in Fig. 3(b) Nonetheless, the benefits of the enhanced approach realize in real-time operation as the re-dispatch cost is lower compared to that yielded by the probabilistic and stochastic approaches as illustrated in Fig. 3(c) As a result, the minimum of the expected total costs is achieved with the enhanced approach as demonstrated by Fig. 3(d).

Increasing the reliability level $\xi$ in the the probabilistic approach may have a positive impact on the performance of the conventional model. However, Table I] shows that this approach never yields the expected cost provided by the proposed model, since the probabilistic approach sets the requirements disregarding their impact on the subsequent op-
TABLE I

COST BREAK-DOWN RESULTING FROM THE IMPLEMENTATION OF A RANGE OF PROBABILISTIC REQUIREMENTS AND ENHANCED REQUIREMENTS.

\begin{tabular}{|c|c|c|c|c|c|c|}
\hline \multirow[t]{2}{*}{ Approach } & \multicolumn{5}{|c|}{$\begin{array}{c}\text { Probabilistic approach } \\
\text { Quantiles } q^{(\underline{\alpha}, \bar{\alpha})} \text { of wind CDF }\end{array}$} & \multirow{2}{*}{$\begin{array}{l}\text { Enhanced } \\
\text { approach }\end{array}$} \\
\hline & $q^{(05 / 95)}$ & $q^{(04 / 96)}$ & $q^{(03 / 97)}$ & $q^{(02 / 98)}$ & $q^{(01 / 99)}$ & \\
\hline Requirements $D^{\mathrm{U} / \mathrm{D}}[\mathrm{MW}]$ & $128 / 89$ & $168 / 91$ & $205 / 93$ & $210 / 94$ & $283 / 169$ & $283 / 43$ \\
\hline Exp. total cost $[\$ 1000]$ & 25.89 & 24.99 & 24.62 & 24.61 & 24.78 & 24.40 \\
\hline - Reserve & 0.69 & 0.84 & 0.99 & 1.01 & 1.70 & 1.24 \\
\hline - Day-ahead & 22.24 & 22.43 & 22.70 & 22.74 & 22.99 & 22.99 \\
\hline - Real-time & 2.96 & 1.72 & 0.93 & 0.86 & 0.88 & 0.18 \\
\hline
\end{tabular}

erations, including potential wind spillage and load shedding. On the contrary, the proposed model finds the optimal tradeoff between reserve procurement and real-time re-dispatch decisions that minimizes the total expected system cost. In this particular case, our model allows more wind curtailment to reduce downward reserve procurement cost.

Regarding the stochastic model, it should be noted that even though reserve requirements are set anticipating the real-time cost, reserve procurement and day-ahead energy schedules are obtained by a co-optimization of these products that is incompatible with the European market structure. As a result, the requirements provided by the stochastic approach lead to larger amounts of load shedding, highlighting that they are practically sub-optimal in a sequential dispatch procedure.

\section{Approximating the stochastic dispatch solution}

We now investigate to what extent the reserve requirements computed with the proposed model are capable of approximating the ideal stochastic solution within the sequential dispatch procedure. To this end, we compare expected daily system cost of three optimization models for different wind power penetration levels, defined as the ratio between the installed capacity of the entire wind power portfolio and the peak load. The first model represents the sequential market clearing (1)- 


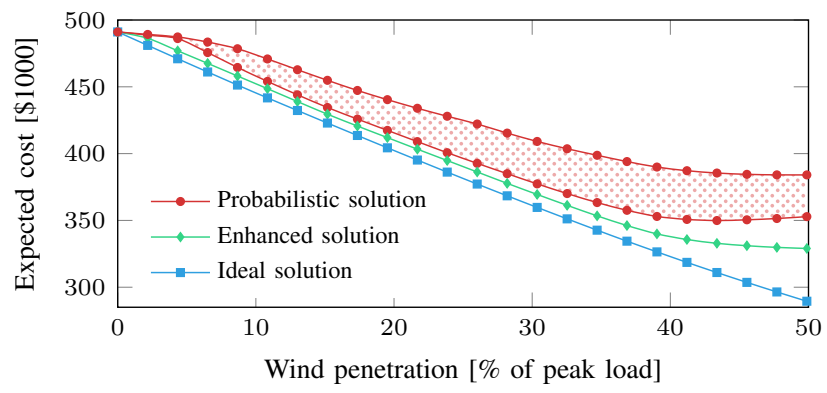

Fig. 4. Expected daily operating cost as a function of wind penetration.

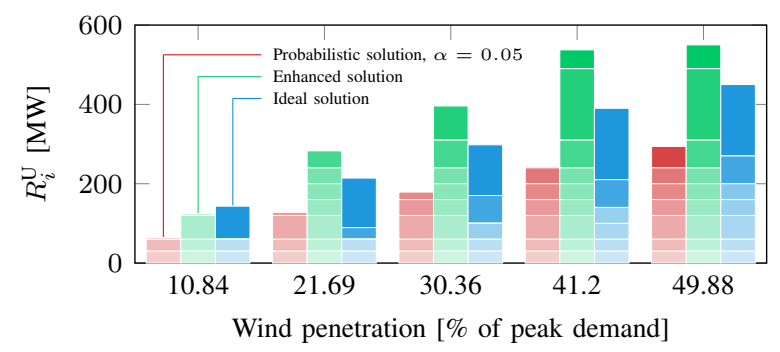

(a) Upward reserve procurement

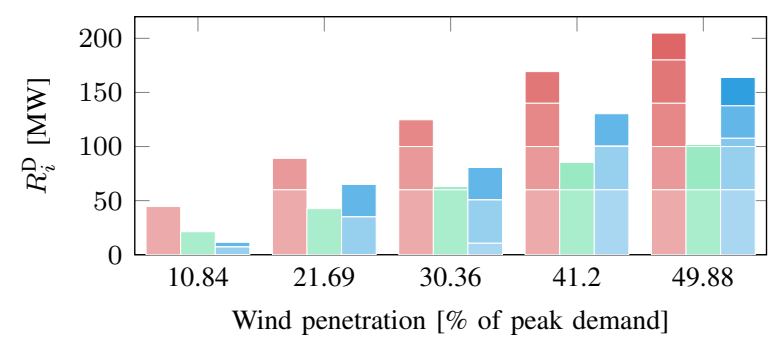

(b) Downward reserve procurement

Fig. 5. Reserve procurement from nine flexible generating units for the peakload hour and different wind penetration levels. Color density ranks generation units according to the reserve capacity price offers.

(3) with reserve requirements computed with the probabilistic approach for a range of reliability levels $\xi \in[0.9,1]$. The second model also follows the sequential market procedure with reserve requirements computed with the proposed model (5). The third one is the stochastic ideal dispatch model (4) that theoretically attains maximum cost-efficiency, and therefore it is used as a lower bound of the expected system cost. It is worth noting the different role that the stochastic dispatch model plays in this part of the case study, compared to the previous Section $\mathrm{V}-\mathrm{B}$. Here, we assume that the solution of the stochastic dispatch model will be implemented as the actual system schedule, presuming that the conventional market setup is replaced with its ideal stochastic counterpart. This is different from the application of the stochastic dispatch model (4) as a reserve-dimensioning approach in Section $\mathrm{V}-\mathrm{B}$, where we considered that all trading floors are settled according to the prevailing European market model.

Figure 4 depicts the daily operating cost as a function of the wind power penetration level for the three models. The setting of the reserve requirements provided by the proposed model always results in a lower expected cost than the implementation of the requirements under the probabilistic approach.

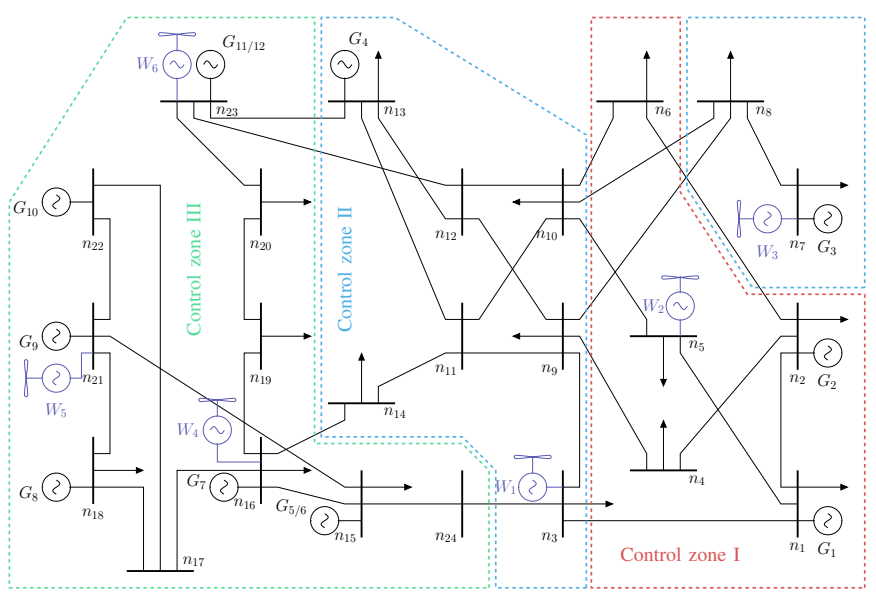

Fig. 6. IEEE 24-Bus reliability test system layout with three reserve control zones.

This figure further indicates that these reserve requirements efficiently approximate the stochastic ideal solution even for a high penetration of wind power.

Figure 5 provides further insights on the difference between the solutions of the three models. Particularly, it shows the procurement of upward and downward reserves from specific flexible units ranked according to their reserve capacity price offers, i.e., from cheap to more expensive units distinguished by increasing color densities. The proposed model controls the trade-off between reserve and real-time costs, ensuring adequate upward reserves to minimize the amount of load shedding and enough downward reserves to prevent wind spillage. In contrast, the probabilistic approach underestimates upward reserve needs, while it overestimates downward reserve requirements.

The enhanced solution for the reserve requirements deviates significantly from the ideal solution given that the stochastic model has more degrees of freedom, i.e., it controls not only the sufficiency of the reserve requirements but also their allocation among the flexible generators. This results in reserve procurement being 'generator-specific' which prevents network congestion within the reserve control area. In attempt to minimize expensive balancing actions, the stochastic model may allocate reserves to more expensive units over cheaper providers, violating the least-cost merit-order principle that is inherent in the conventional market design. As a consequence, the requirement imposed in our enhanced approach to respect the merit-order principle in the reserve capacity and day-ahead markets restricts the degree of approximation of the stochastic solution.

\section{Optimal zonal reserve requirements allocation}

We now consider the optimal reserve dimensioning in a multi-zone setting. For this purpose, the IEEE 24-Bus system is split into three reserve control zones as depicted in Fig. 6 This zonal layout corresponds to the one proposed in [11]. In each control zone there are at least one wind power unit with capacity of $100 \mathrm{MW}$ and at least two flexible generation units. Unlike in the previous instance, the requirements computed with the probabilistic approach are now set for each reserve control zone independently considering the distribution of 


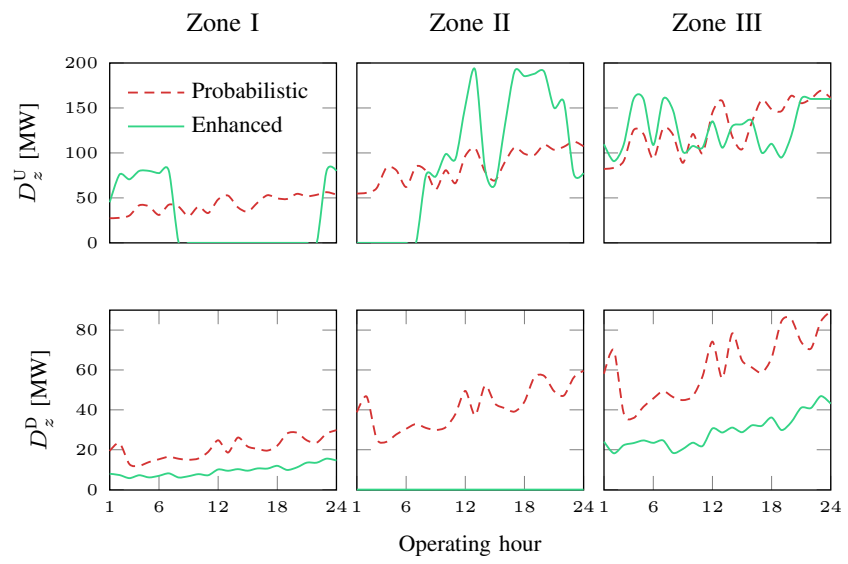

Fig. 7. 24-hour profiles of probabilistic and enhanced reserve requirements in three control zones.

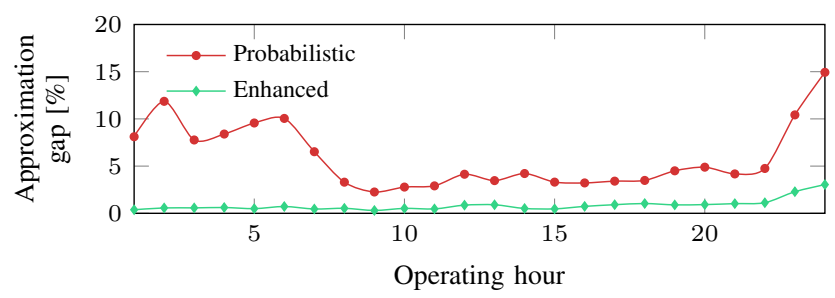

Fig. 8. Approximation gap of the sequential market with probabilistic and enhanced reserve requirements compared to stochastic dispatch.

wind power production of each zone. The reliability level $\xi$ is set to 0.98 .

The resulting allocation for upward and downward reserve requirements among control zones is summarized in Fig. 7 , indicating that the probabilistic approach sets the reserve needs proportionally to the amount of stochastic in-feed in the respective control zone. On the other hand, the proposed model defines the requirements considering not only the zonal wind power in-feed, but also the cost implications of procuring reserve in a specific zone. As a result, the model finds it more efficient to constantly procure upward reserve from the third zone and obtain the remaining upward reserve that is needed either from the first or the second zone depending on the operating hour. In addition, this reserve allocation indicates that it is never optimal to procure downward reserve from the second zone in terms of expected system cost.

This optimal reserve allocation among control zones is supported by the approximation gap depicted in Fig. 8, showing the relative cost difference of the sequential market with respect to the ideal solution. The requirements provided by the proposed model efficiently approximate the ideal solution with nearly zero gap over the first operating hours, and this gap remains relatively small for the subsequent hours as opposed to the large gap when probabilistic requirements are used. The definition of multiple control zones allows to set enhanced reserve requirements that are closer to the 'generator-specific' reserve allocation of the stochastic model. Indeed, compared to the single-zone setup in section $\mathrm{V}-\mathrm{B}$, the operating cost reduces by $2.5 \%$, from $\$ 24,408$ to $\$ 24,034$, after the definition of three control zones.

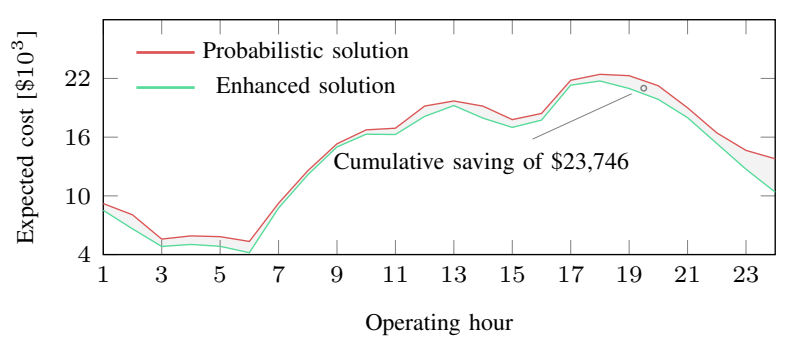

(a) Reliability level $\xi=98 \%$

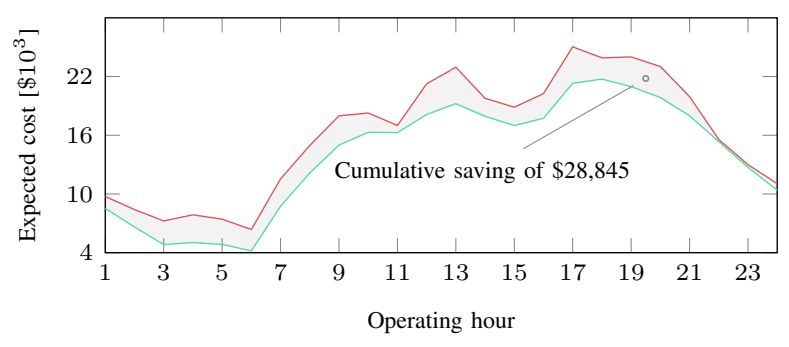

(b) Reliability level $\xi=90 \%$

Fig. 9. Expected operating cost yielded by the implementation of the probabilistic and enhanced reserve requirements in the conventional marketclearing problem (1)-3] including the unit commitment constraints $9 \mathrm{a}-\mathrm{-9 \textrm {g }}$.

\section{E. Assessing enhanced reserve requirements in the presence} of non-convexities

To assess the performance for the proposed reserve quantification model as a proxy model for the power markets with a more comprehensive and non-convex representation of technical constraints, we use the enhanced reserve requirements provided by the proposed model (5) as inputs to the sequential market-clearing problem (1)-(3) with unit commitment and ramping constraints integrated in the day-ahead auction as explained in Appendix A

Figure 9 shows the hourly profile of expected operating system cost resulting from the implementation of the enhanced requirements in the system with full representation of the technical constraints. This profile is compared against those obtained by setting probabilistic reserve requirements with reliability levels of $98 \%$ and $90 \%$. The reserve requirements provided by the proposed model always attain better cost efficiency than the probabilistic requirements, even though the proposed model does not account for the whole set of technical limits of power plants. In the first case in Fig 9 (a), the model allows savings of $\$ 23,746$ that nearly equal to the cost of peakhour operation, and it allows even larger savings of $\$ 28,845$ in the second case in Fig9 (b).

\section{F. Application to the IEEE-96 RTS}

We now consider the modernized version of the IEEE-96 RTS Test System proposed in [38] to assess the scalability of the proposed model. The test system includes three control zones interconnected by six tie-lines. The system demand follows a 24-hour profile with a peak load of $7.5 \mathrm{GW}$. The conventional generation is represented by 6 nuclear power plants serving the base load, 3 coal power plants that offer $40 \%$ of their capacities for the reserve needs, and 87 gasfired power plants offering $100 \%$ of their capacities to the reserve procurement auction. The reserve offering prices of 
TABLE II

CPU PERFORMANCE OF THE BENDER'S ALGORITHM.

\begin{tabular}{c|ccc}
\hline Wind penetration [\%] & 13.8 & 23.0 & 36.8 \\
CPU time [min] & 32.1 & 33.5 & 58.6 \\
\hline
\end{tabular}

TABLE III

DAILY OPERATING COST WITH PROBABILISTIC AND ENHANCED ZONAL RESERVE REQUIREMENTS IN COMPARISON WITH THE STOCHASTIC IDEAL SOLUTION [\$1000].

\begin{tabular}{ccccc}
\hline Wind penetration & \multicolumn{2}{c}{$\begin{array}{c}\text { Probabilistic } \\
\text { solution }\end{array}$} & $\begin{array}{c}\text { Enhanced } \\
\text { solution }\end{array}$ & $\begin{array}{c}\text { Ideal } \\
\text { solution }\end{array}$ \\
\cline { 2 - 3 } & $\xi=90 \%$ & $\xi=98 \%$ & & \\
\hline 13.8 & $1,912.4$ & $1,888.8$ & $1,877.3$ & $1,850.0$ \\
23.0 & $1,760.8$ & $1,719,3$ & $1,700.8$ & $1,660.5$ \\
36.8 & $1,550.7$ & $1,482.3$ & $1,446.0$ & $1,402.8$ \\
\hline
\end{tabular}

flexible units are set to $25 \%$ of marginal production cost for both upward and downward reserve needs. There are 19 wind farms distributed among the control zones with the overall capacity of $2.76 \mathrm{GW}$. Their stochastic output is described by 100 equiprobable scenarios obtained from [39]. The input data and the corresponding GAMS codes are provided in the electronic companion of the paper [37].

The test case is solved for wind penetration levels of $13.8 \%$, $23.0 \%$, and $36.8 \%$ of the peak-hour load by implementing the multicut Bender's algorithm explained in Section IV] The tolerance of the algorithm is set to $0.02 \%$ requiring three to eight iterations depending on the operating hour. The resulting CPU time is reported in Table II The CPU time in all three cases is kept below one hour allowing timely day-ahead planning with the proposed model. It is worth mentioning that the CPU time can be reduced at the expense of a marginal deviation from the global optimum with higher tolerance.

The daily operating cost resulting from the implementation of the enhanced zonal reserve requirements computed by the proposed model is always lower than those provided by the probabilistic approach with reliability levels of $90 \%$ and $98 \%$, as demonstrated in Table III The difference in operating cost is explained by the anticipated cost of procuring upward and downward reserves from a specific control zone, while the probabilistic requirements are solely obtained proportionally to the amount of stochastic in-feed in control zones. As a result, the relative cost savings provided by the model increases with the wind penetration level and ranges between $0.6 \%$ and $7.2 \%$. Further cost savings towards the ideal solution provided by the stochastic model is limited due to the enforced merit order in both reserve and day-ahead markets. Finally, Table IV] illustrates the economic benefit that the proposed model yields as a proxy for the system with the full network representation and technical constraints of power plants described in Appendix A The results show that in spite of the incomplete description of technical constraints in the lower level of the proposed bilevel model, it still provides a feasible input with a sensible cost reduction for the markets with non-convexities. The economic benefit provided by the model ranges from $0.5 \%$ to $1.6 \%$. Moreover, the proposed approach further outperforms the probabilistic one for the largest wind penetration level, where the overestimated requirements provided by the probabilistic approach lead to a reserve schedule that results in an infeasible
TABLE IV

DAILY OPERATING COST WITH PROBABILISTIC AND ENHANCED ZONAL RESERVE REQUIREMENTS WITH FULL REPRESENTATION OF TECHNICAL CONSTRAINTS $[\$ 1000]$.

\begin{tabular}{cccc}
\hline \multirow{2}{*}{$\begin{array}{c}\text { Wind penetration } \\
{[\%]}\end{array}$} & \multicolumn{2}{c}{$\begin{array}{c}\text { Probabilistic } \\
\text { solution }\end{array}$} & \multirow{2}{*}{$\begin{array}{c}\text { Enhanced } \\
\text { solution }\end{array}$} \\
\cline { 2 - 3 } & $\xi=90 \%$ & $\xi=98 \%$ & \\
\hline 13.8 & $2,072.2$ & $2,073.4$ & $2,061.5$ \\
23.0 & $1,947.9$ & $1,949.1$ & $1,928.6$ \\
36.8 & $1,764.4$ & infeas. & $1,735.9$ \\
\hline
\end{tabular}

day-ahead operation.

\section{CONCLUSION}

This paper considers the optimal setting of reserve requirements in a European market framework. We propose a new method to quantify reserve needs that brings the sequence of the reserve, day-ahead and real-time markets closer to the ideal stochastic energy and reserves co-optimization model in terms of total expected cost. The proposed model is formulated as a stochastic bilevel problem, which is eventually recast as a MILP problem. To reduce the computational burden of this model, we apply an iterative solution approach based on the multi-cut Bender's decomposition algorithm.

Our numerical studies demonstrate the benefit of properly setting reserve requirements. Our reserve quantification model outperforms both the probabilistic and the stochastic reserve setting approaches due to its preemptive ability to anticipate the impact of day-ahead decisions on the real-time operation, while taking into account the actual market structure. Considering the increasing penetration of stochastic power producers, we show that the reserve requirements provided by the proposed model take the expected system operating cost closer to that given by the ideal energy and reserve co-optimization model, but the degree of this approximation is limited due to the sequential scheduling of reserve and energy in European electricity markets. However, our analysis further indicates that the definition of multiple reserve control zones allows for a more efficient spatial allocation of reserves, which reduces the approximation gap with respect to the ideal stochastic model. Finally, the efficiency of the proposed reserve dimensioning model was tested against market designs whose clearing process explicitly account for inter-temporal and non-convex constraints, i.e. ramping limits and unit commitment constraints. Even though the proposed model does not account for the whole set of technical constraints of such markets, the enhanced reserve requirements still bring the cost of sequential market operation closer to the stochastic ideal, highlighting the importance of the intertemporal coordination between the three trading floors through the intelligent setting of reserve needs.

Future research may focus on the consideration of the tight relaxations of the unit commitment constraints to achieve better approximations for the case of non-convex market designs, and the corresponding tuning of the Bender's decomposition algorithm to better cope with the intertemporal constraints. 


\section{APPENDIX}

\section{A. Incorporation of unit commitment and ramping constraints}

In contrast to the prevailing approach of the European market design, other electricity markets, e.g., the majority of US markets, explicitly model unit commitment constraints and thermal limits of power plants in the market-clearing problem. To assess the performance of the proposed reserve quantification model in markets with unit commitment constraints, the following set of constraints are integrated in the day-ahead market-clearing problem:

$$
\begin{aligned}
& u_{i t} \underline{P}_{i} \leq P_{i t}^{\mathrm{C}} \leq u_{i t} \bar{P}_{i}, \forall i \in I, \forall t \in T, \\
& S U_{i t} \geq C_{i}^{\mathrm{SU}}\left(u_{i t}-u_{i(t-1)}\right), \forall i \in I, \forall t>1, \\
& S U_{i t} \geq C_{i}^{\mathrm{SU}}\left(u_{i t}-u_{i}^{0}\right), \forall i \in I, t=1, \\
& P_{i t}^{\mathrm{C}}-P_{i(t-1)}^{\mathrm{C}} \leq R_{i}^{+}, \forall i \in I, \forall t>1, \\
& P_{i t}^{\mathrm{C}}-P_{i}^{\mathrm{C}, 0} \leq R_{i}^{+}, \forall i \in I, t=1, \\
& P_{i(t-1)}^{\mathrm{C}}-P_{i t}^{\mathrm{C}} \leq R_{i}^{-}, \forall i \in I, \forall t>1, \\
& P_{i}^{\mathrm{C}, 0}-P_{i t}^{\mathrm{C}} \leq R_{i}^{-}, \forall i \in I, t=1,
\end{aligned}
$$

where $t \in T$ is the set of operating hours, $C_{i}^{\mathrm{SU}}$ is a startup cost of unit $i, R_{i}^{+}$and $R_{i}^{-}$are the ramp-up and rampdown limits, $\underline{P}_{i}$ is a minimum power output limit, and $P_{i}^{\mathrm{C}, 0}$ and $u_{i}^{0}$ are the initial power output and commitment status of unit $i$. The set of decision variables of the original problem is supplemented with variable $u_{i t} \in\{0,1\}$ that denotes the commitment status of generating units, and variable $S U_{i t}$ that computes the cost induced by the start-up of generating units. Now, the generating limits of each unit are additionally enforced by commitment decisions of the system operator by 9a). Binary logic is controlled by (9b) and (9c) and activated by augmenting $S U_{i t}$ into the original objective function of problem (2). The ramp limits of generators are accounted for through (9d)-(9g).

\section{REFERENCES}

[1] T. Aigner, S. Jaehnert, G. L. Doorman, and T. Gjengedal, "The effect of large-scale wind power on system balancing in Northern Europe," IEEE Trans. Sustain. Energy, vol. 3, no. 4, pp. 751-759, 2012.

[2] J. M. Morales, A. J. Conejo, K. Liu, and J. Zhong, "Pricing electricity in pools with wind producers," IEEE Trans. Power Syst., vol. 27, no. 3, pp. 1366-1376, 2012.

[3] F. Bouffard, F. D. Galiana, and A. J. Conejo, "Market-clearing with stochastic security-part I: formulation," IEEE Trans. Power Syst., vol. 20, no. 4, pp. 1818-1826, 2005.

[4] A. Papavasiliou, S. S. Oren, and B. Rountree, "Applying high performance computing to transmission-constrained stochastic unit commitment for renewable energy integration," IEEE Trans. Power Syst., vol. 30, no. 3, pp. 1109-1120, 2015.

[5] A. Tuohy, P. Meibom, E. Denny, and M. O'Malley, "Unit commitment for systems with significant wind penetration," IEEE Trans. Power Syst., vol. 24, no. 2, pp. 592-601, 2009.

[6] J. Wang, M. Shahidehpour, and Z. Li, "Security-constrained unit commitment with volatile wind power generation," IEEE Trans. Power Syst. vol. 23, no. 3, pp. 1319-1327, 2008.

[7] V. M. Zavala, K. Kim, M. Anitescu, and J. Birge, "A stochastic electricity market clearing formulation with consistent pricing properties," Oper. Res., vol. 65, no. 3, pp. 557-576, 2017.

[8] J. M. Morales, M. Zugno, S. Pineda, and P. Pinson, "Electricity market clearing with improved scheduling of stochastic production," Eur. J. Oper. Res., vol. 235, no. 3, pp. 765-774, 2014.

[9] J. Kazempour, P. Pinson, and B. F. Hobbs, "A stochastic market design with revenue adequacy and cost recovery by scenario: Benefits and costs," IEEE Trans. Power Syst., vol. 33, no. 4, pp. 3531-3545, 2018.
[10] M. Sarfati, M. R. Hesamzadeh, D. R. Biggar, and R. Baldick, "Probabilistic pricing of ramp service in power systems with wind and solar generation," Renewable and Sustainable Energy Reviews, vol. 90, pp. $851-862,2018$

[11] T. V. Jensen, J. Kazempour, and P. Pinson, "Cost-optimal ATCs in zonal electricity markets,” IEEE Trans. Power Syst., vol. 33, no. 4, pp. 36243633, 2018.

[12] B. Wang and B. F. Hobbs, "Flexiramp market design for real-time operations: Can it approach the stochastic optimization ideal?" in 2013 IEEE PESGM, 2013, pp. 1-5.

[13] W. W. Hogan, "Electricity scarcity pricing through operating reserves," EEEP, vol. 2, no. 2, pp. 65-86, 2013.

[14] A. Papavasiliou and Y. Smeers, "Remuneration of flexibility using operating reserve demand curves: A case study of Belgium." Energy Journal, vol. 38, no. 6, 2017.

[15] Y. Rebours and D. Kirschen, "A survey of definitions and specifications of reserve services," Tech. Rep., 2005.

[16] "Emergency Operations," PJM Manual, Tech. Rep., 2011. [Online]. Available: http://www.pjm.com/ /media/documents/manuals/ archive/m13/m13v46-emergency-operations-11-16-2011.ashx

[17] G. Strbac, A. Shakoor, M. Black, D. Pudjianto, and T. Bopp, "Impact of wind generation on the operation and development of the UK electricity systems," Electr. Pow. Syst. Res., vol. 77, no. 9, pp. 1214-1227, 2007.

[18] D. Lee and R. Baldick, "Analyzing the variability of wind power output through the power spectral density," in 2012 IEEE PESGM, 2012, pp. $1-8$.

[19] R. Doherty and M. O'Malley, "A new approach to quantify reserve demand in systems with significant installed wind capacity," IEEE Trans. Power Syst., vol. 20, no. 2, pp. 587-595, 2005.

[20] Y. V. Makarov, P. V. Etingov, J. Ma, Z. Huang, and K. Subbarao, "Incorporating uncertainty of wind power generation forecast into power system operation, dispatch, and unit commitment procedures," IEEE Trans. Sustain. Energy, vol. 2, no. 4, pp. 433-442, 2011.

[21] Y. Dvorkin, D. S. Kirschen, and M. A. Ortega-Vazquez, "Assessing flexibility requirements in power systems," IET GENER. TRANSM. DIS., vol. 8, no. 11, pp. 1820-1830, 2014.

[22] H. Nosair and F. Bouffard, "Flexibility envelopes for power system operational planning," IEEE Trans. Sustain. Energy, vol. 6, no. 3, pp. 800-809, 2015.

[23] — , "Economic dispatch under uncertainty: The probabilistic envelopes approach," IEEE Trans. Power Syst., vol. 32, no. 3, pp. 17011710, 2017.

[24] M. Lange, "On the uncertainty of wind power predictionsanalysis of the forecast accuracy and statistical distribution of errors," J. Sol. Energy Eng., vol. 127, no. 2, pp. 177-184, 2005.

[25] M. A. Matos and R. J. Bessa, "Setting the operating reserve using probabilistic wind power forecasts," IEEE Trans. Power Syst., vol. 26, no. 2, pp. 594-603, 2011

[26] H. Holttinen, M. Milligan, E. Ela, N. Menemenlis, J. Dobschinski, B. Rawn, R. J. Bessa, D. Flynn, E. Gomez-Lazaro, and N. K. Detlefsen, "Methodologies to determine operating reserves due to increased wind power," IEEE Trans. Sustain. Energy, vol. 3, no. 4, pp. 713-723, 2012.

[27] P. N. Biskas, D. I. Chatzigiannis, and A. G. Bakirtzis, "European electricity market integration with mixed market designs-part I: Formulation," IEEE Trans. Power Syst., vol. 29, no. 1, pp. 458-465, 2014.

[28] S. Kasina, "Essays on unit commitment and interregional cooperation in transmission planning," 2017, Ph.D. thesis.

[29] J. Kazempour and B. F. Hobbs, "Value of flexible resources, virtual bidding, and self-scheduling in two-settlement electricity markets with wind generation-part I: Principles and competitive model," IEEE Trans. Power Syst., vol. 33, no. 1, pp. 749-759, 2018.

[30] C. Ruiz, A. J. Conejo, and D. J. Bertsimas, "Revealing rival marginal offer prices via inverse optimization," IEEE Trans. Power Syst., vol. 28 , no. 3, pp. 3056-3064, 2013.

[31] L. Mitridati and P. Pinson, "A bayesian inference approach to unveil supply curves in electricity markets," IEEE Trans. Power Syst., vol. 33, no. 3, 2017.

[32] D. Pozo, E. Sauma, and J. Contreras, "Basic theoretical foundations and insights on bilevel models and their applications to power systems," Ann. Oper. Res., pp. 1-32, 2017.

[33] A. J. Conejo, E. Castillo, R. Minguez, and R. Garcia-Bertrand, Decomposition techniques in mathematical programming: engineering and science applications. Springer Science \& Business Media, 2006.

[34] C. Ordoudis, P. Pinson, J. M. Morales, and M. Zugno, "An updated version of the IEEE RTS 24-Bus system for electricity market and power system operation studies," Technical University of Denmark, 2016. 
[35] D. J. Swider and C. Weber, "Bidding under price uncertainty in multiunit pay-as-bid procurement auctions for power systems reserve," Eur. J. Oper. Res., vol. 181, no. 3, pp. 1297 - 1308, 2007.

[36] M. A. Plazas, A. J. Conejo, and F. J. Prieto, "Multimarket optimal bidding for a power producer," IEEE Trans. Power Syst., vol. 20, no. 4 , pp. 2041-2050, 2005.

[37] V. Dvorkin, S. Delikaraoglou, and J. M. Morales. (2018) Online appendix of the paper "Setting reserve requirements to approximate the efficiency of the stochastic dispatch". [Online]. Available: https://doi.org/10.5281/zenodo.1408881

[38] H. Pandzic, Y. Dvorkin, T. Qiu, Y. Wang, and D. Kirschen, "Unit commitment under uncertainty - GAMS models," Library of the Renewable Energy Analysis Lab (REAL), University of Washington, Seattle, USA. [Online]. Available: http://www.ee.washington.edu/research/real/gams_code.html

[39] P. Pinson, "Wind energy: Forecasting challenges for its operational management," Stat. Sci., pp. 564-585, 2013.

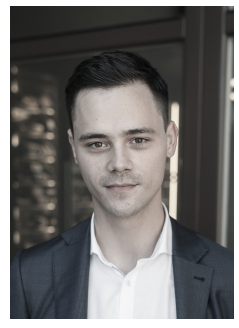

Vladimir Dvorkin Jr. (S'18) received the B.S. degree in electrical engineering from the Moscow Power Engineering Institute, Russia, in 2012, the M.Sc. degree in Economics from the Higher School of Economics, Russia, in 2014, and the M.Sc. degree in Sustainable Energy from the Technical University of Denmark in 2017. Currently he is pursuing the Ph.D. degree with the Department of Electrical Engineering, Center for Electric Power and Energy, Technical University of Denmark.

His research interests include economics, game theory, optimization, and their applications to power systems and electricity markets.

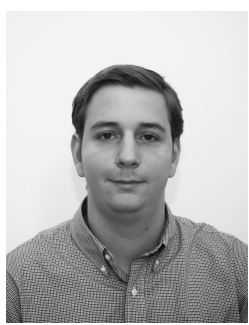

Stefanos Delikaraoglou (S'14 - M18) received the Dipl.-Eng. degree from the School of Mechanical Engineering, National Technical University of Athens, Greece, in 2010 and the M.Sc. degree in Sustainable Energy from the Technical University of Denmark in 2012. He holds a Ph.D. degree awarded in 2016 by the Department Electrical Engineering at the Technical University of Denmark. He is currently a Postdoctoral Fellow with the EEH-Power Systems Laboratory at the Swiss Federal Institute of Technology (ETH), Zurich, Switzerland.

His research interests include energy markets and multi-energy systems modeling, decision-making under uncertainty, equilibrium models and hierarchical optimization.

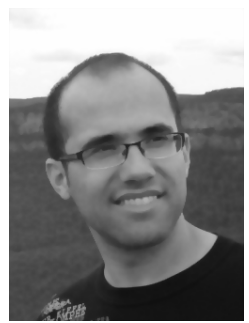

Juan M. Morales (S'07-M'11-SM'16) received the Ingeniero Industrial degree from the University of Málaga, Málaga, Spain, in 2006, and a Ph.D. degree in Electrical Engineering from the University of Castilla-La Mancha, Ciudad Real, Spain, in 2010. $\mathrm{He}$ is currently an associate professor in the Department of Applied Mathematics at the University of Málaga in Spain.

His research interests are in the fields of power systems economics, operations and planning; energy analytics and optimization; smart grids; decisionmaking under uncertainty, and electricity markets. 